\title{
Atuação da Estratégia Saúde da Família e do Núcleo de Apoio à Saúde da Família no uso racional de medicamentos em Rio Fortuna, em Santa Catarina
}

\author{
Core of the Family Health Strategy and of the Family Health Support in the \\ rational use of drugs in Rio Fortuna, Santa Catarina \\ Actuación de la Estrategia del Núcleo de Apoyo a la Salud de la Familia en el \\ uso racional de medicamentos en Rio Fortuna, en Santa Catarina \\ Fabio Tetuo Omomo ${ }^{1 *}$, Tiago Michels Bechtold ${ }^{2}$
}

Palavras-chave: Medicamentos Polifármacos Assistência Domiciliar Saúde da Família

\section{Resumo}

A atenção farmacêutica tem sido foco de grande preocupação, principalmente no que diz respeito ao uso correto de medicamentos, automedicação, intoxicações, interações medicamentosas e efeitos adversos danosos à população. 0 presente trabalho teve como objetivo geral avaliar o uso racional de medicamentos junto à população de abrangência das equipes de saúde da família do município de Rio Fortuna, em Santa Catarina. Os objetivos específicos foram: informar a população sobre o problema do uso e abuso de medicamentos; avaliar a aceitação do debate sobre o tema; identificar polifarmácia domiciliar e o risco de abuso ou intoxicação medicamentosa, bem como servir de educação permanente em saúde aos trabalhadores do setor, e recolher medicamentos ociosos. Este é um estudo descritivo, transversal, quantiqualitativo, com dados coletados em inquérito domiciliar realizado por Agentes Comunitários de Saúde, além da realização de metodologia de educação permanente em saúde por meio de palestras e orientações do uso racional de medicamentos aplicados tanto para a equipe de saúde quanto à população. Foram realizadas 1.054 visitas a domicílios, sendo identificadas 185 famílias de risco quanto à presença de polifarmácia domiciliar. A média do consumo de medicamentos na população geral foi de dois a três comprimidos/habitante/dia, enquanto que, nas famílias em que havia polifarmácias, o consumo passou de nove a dez comprimidos por morador, por dia. Foram realizadas também 13 palestras educativas, ao final das quais aplicouse questionário avaliativo da importância deste trabalho, no qual 98\% dos participantes acharam importante a sua realização, 94\% manifestaram que se sentiram mais informados a respeito do uso racional de medicamentos e 50\% já praticaram a automedicação. Por meio deste trabalho, pode ter-se a percepção real da necessidade que a população tem do tratamento medicamentoso, muitas vezes sem orientação profissional e o limitado conhecimento dos riscos da automedicação e dos perigos das interações medicamentosas. Além disso, percebe-se que a atuação multiprofissional na atenção em saúde é essencial para o constante aprimoramento do cuidado da saúde e o entendimento de que a educação permanente em saúde, tanto dos profissionais quanto da população e da gestão setorial, pode promover grandes mudanças no paradigma assistencial.

Secretaria Municipal de Saúde de Rio Fortuna. fabiotomomo@yahoo.com.br

2Secretaria Municipal de Saúde de Rio Fortuna. farmacia@riofortuna.sc.gov.br

*Autor correspondente.

Fonte de financiamento: Secretaria Municipal de Saúde de Rio Fortuna - PSF/Nasf

Conflito de interesses: declararam não haver

Recebido em: 16/06/2011

Aprovado em: 06/01/2012 


\section{Keywords: Abstract \\ Drugs}

Polypharmacy

Home Nursing

Family Health

Pharmaceutical attention has been the focus of major concern, especially regarding the correct use of medicine, self-medication, intoxication, drug interactions, and adverse effects, which are harmful to the population. This paper had as its general purpose to evaluate the rational use of medicine together with the population of family health teams from the municipality of Rio Fortuna, in Santa Catarina State. The specific purposes were: to inform people about the issue of using and abusing of drugs; to evaluate the acceptability of the debate on the topic; to identify the risk of polypharmacy and household abuse or drug poisoning as well as to serve as a permanent education for health industry workers; and to collect stranded medicines. This is a descriptive, cross-sectional and quanti-qualitatively study, with data collected in a household survey carried out by community health agents, in addition to the achievement of permanent education methodology in health through lectures and guidelines of the rational use of medicines applied both to health staff and the population. 1,054 households were carried out, and 185 families were identified with risks as to the presence of domiciliary polypharmacy. The average consumption of medicine in the general population was from two to three tablets/ resident/day, while in families in which there were polypharmacies, consumption, it rose from nine to ten pills per resident per day. Thirteen educational lectures were held; at the end, an assessment questionnaire was applied on the importance of this paper, in which $98 \%$ of the participants thought important their attainment, $94 \%$ expressed that they felt more informed regarding the rational use of medicines, and $50 \%$ had already practiced self-medication. Through this study, the real perception of the need that the population has of drug treatment can be seen, often without professional guidance, limited knowledge of risks of self-medication, and dangers of medicine interaction. In addition, it could be seen that acting in multiprofessional health is essential for the constant improvement of health care and understanding that permanent education of both health professionals, the population and the sector management may promote great changes in the assistive paradigm.

Palabras clave:

Medicamentos

Polifarmacia

Atención Domiciliaria de Salud Salud de la Familia

\section{Resumen}

Atención farmacéutica ha sido objeto de gran preocupación, especialmente en relación con el uso correcto de las drogas, automedicación, intoxicación, interacciones medicamentosas y efectos adversos nocivos para la población. Este trabajo tuvo como objetivo general evaluar el uso racional de medicamentos a la población de la amplitud de los equipos de salud familiar de la municipalidad de Río Fortuna, en Santa Catarina. Las metas específicas fueron: informar al público sobre el problema del uso y abuso de drogas; evaluar la aceptabilidad del debate sobre el tema; identificar el riesgo de abuso polypharmacy y hogar o intoxicación por drogas, así como servir de una educación permanente de los trabajadores del sector de salud y recoger medicamentos varados. Esto es un estudio descriptivo de corte transversal, quanti-cualitativamente, con datos recogidos en la encuesta de hogares realizadas por agentes de salud comunitarios, además de la realización de la metodología de la educación permanente en salud a través de conferencias y directrices de uso racional de medicamentos aplicados tanto al personal de salud y a la población. Las visitas se realizaron en 1.054 hogares y 185 familias fueron identificadas con riesgos sobre la presencia de polypharmacy domiciliaria. El consumo promedio de medicamentos en la población general fue de dos a tres tabletas/habitante/día, mientras que en las familias donde hubo polifarmácias, consumo pasó de nove a diez píldoras por habitante al día. Trece conferencias educativas se celebraron también, en el fin se aplicó un cuestionario evaluativo de la importancia de este trabajo, en el cual el 98\% de los participantes consideró importante para su consecución, el 94\% expresó que se sentían más informados acerca del uso racional de medicamentos y el $50 \%$ practica ya la automedicación. A través de este trabajo, puede se tener la percepción real de la necesidad de que la población tiene de tratamiento de drogas, a menudo sin orientación profesional y el limitado conocimiento de los riesgos de la automedicación y los peligros de las interacciones de drogas. Además, se da cuenta que actuar en salud multiprofesional es esencial para la mejora constante de la atención de la salud y el entendimiento de que la educación permanente de profesionales de la salud y la población y administración sectorial puede promover grandes cambios en el paradigma de la asistencia.

\section{Introdução}

À medida que a população cresce e envelhece, aumentam as necessidades por atendimento em saúde e, em consequência, a demanda por medicamentos. No Brasil, desde a implantação da Política Nacional de Medicamentos (Portaria GM 3.916/98), tem-se buscado atender às prioridades locais e disponibilizar medicamentos da farmácia básica, principalmente à população com maior dificuldade de acesso. Isso tem o propósito de garantir a necessária segurança, eficácia e qualidade dos medicamentos, a promoção do uso racional e o acesso da população àqueles considerados essenciais. Por essa nova política de descen- tralização das ações em saúde, o Ministério da Saúde passa a transferir aos municípios recursos financeiros ou medicamentos e, principalmente, a responsabilidade da assistência farmacêutica básica à aquisição de medicamentos essenciais de saúde mental, de alto custo, e dos programas estratégicos (AIDS, hanseníase, tuberculose etc. $)^{1,2}$.

Documentos técnicos do Ministério da Saúde descrevem que o consumo de medicamentos é influenciado também pelos indicadores demográficos, os quais têm demonstrado clara tendência de aumento na expectativa de vida ao nascer. O processo de envelhecimento populacional interfere, sobretudo, na demanda por medicamentos destinados ao tratamento de doenças crônicas não-trans- 
missíveis, além de novos procedimentos terapêuticos com a utilização de produtos de alto custo. Igualmente, adquire especial relevância o aumento da demanda por medicamentos de uso contínuo, como é o caso daqueles utilizados no tratamento de doenças cardiovasculares, reumáticas e no controle do diabetes ${ }^{1,3}$.

No entanto, se, por um lado, houve melhoras no acesso aos medicamentos, por outro, o consumo progressivamente maior, por parte da população, tem trazido preocupação às equipes de saúde. A política farmacêutica nacional, principalmente a da Farmácia Básica, ao mesmo tempo em que possibilita medicamentos essenciais de forma gratuita à população, gera necessidade cada vez maior de aquisição de insumos pela administração municipal e, em muitos casos, consumo exagerado, abusivo ou situações de intoxicação medicamentosa.

Dados da Organização Mundial de Saúde (OMS) apontam que $15 \%$ da população mundial consomem mais de $90 \%$ da produção farmacêutica e 25 a $70 \%$ do gasto em saúde nos países em desenvolvimento correspondem a medicamentos, enquanto que, em países desenvolvidos, esses gastos são menores de 15\%. Cerca de 50 a $70 \%$ das consultas médicas geram prescrição medicamentosa e $50 \%$ dos medicamentos são prescritos, dispensados ou usados inadequadamente. Cerca de $75 \%$ das prescrições com antibióticos são errôneas. Somente $50 \%$ dos pacientes, em média, tomam corretamente seus medicamentos 5 .

Quanto ao estoque de medicamentos nos domicílios e polifarmácia caseira, estudos têm demonstrado que a média de medicamentos por domicílio chega a 8,4 por família, sendo mais frequente a presença de analgésicos/antirreumáticos, descongestionantes, antibacterianos para uso sistêmico e antiácidos ${ }^{6-8}$.

Algumas pessoas estão mais predispostas a interações e aos efeitos medicamentosos adversos, tais como: crianças, idosos, gestantes, portadores de insuficiência renal e hepática, doenças cardíacas e respiratórias, diabetes, hipotireoidismo e muitas outras ${ }^{9-13}$.

Dados estatísticos têm demonstrado que 3 a $4 \%$ das hospitalizações de idosos e $4 \%$ do total de óbitos estão associados à intoxicação medicamentosa. Estatísticas do Centro de Informações Toxicológicas do Estado de Santa Catarina (CIT/SC) têm registrado os medicamentos como a segunda causa mais frequente de situações de intoxicação, ficando atrás somente dos acidentes com animais peçonhentos ${ }^{14}$.

Desta forma, é necessário desenvolver trabalhos avaliativos a respeito do uso racional de medicamentos. Deve-se haver definição de alguns conceitos como "uso racional de medicamentos", "polifarmácia domiciliar", "efeitos adversos" e "intoxicação medicamentosa", além da orientação junto à equipe de saúde e à população sobre o tema, com levantamentos de grupos de risco e intervenção para minimizar os efeitos das interações medicamentosas adversas e os possíveis danos à saúde da população.

Este trabalho teve como objetivo geral desenvolver atividades de uso racional de medicamentos junto à população de abrangência das duas equipes de saúde da família do município de Rio Fortuna, em Santa Catarina. Teve como objetivos específicos informar a população sobre o problema do uso e abuso de medicamentos; avaliar a aceitação do debate sobre o tema; identificar, nos domicílios, por meio de questionário-enquete da visita do agente comunitário de saúde, da existência de polifarmácia domiciliar, do risco da automedicação e de abuso ou intoxicação medicamentosa; bem como servir de educação permanente em saúde aos trabalhadores do setor. $\mathrm{Na}$ oportunidade das visitas, serão recolhidos medicamentos considerados sem uso e aqueles que tenham data de validade vencida ou que possuam qualidade duvidosa. Por fim, as pessoas identificadas como de risco por uso de cinco ou mais medicamentos por dia serão avaliadas clinicamente sobre danos de função hepática e renal, bem como riscos de interação medicamentosa dos fármacos de uso individual.

\section{Metodologia}

O presente trabalho consiste em um estudo descritivo transversal, de base populacional, realizado por meio da análise de dados quantiqualitativos coletados pela equipe de Saúde da Família em três momentos distintos: questionário de interesse e aceitação da pesquisa junto aos participantes das palestras de apresentação do trabalho, enquete domiciliar sobre a existência de polifarmácias e avaliação clínica dos indivíduos do grupo de risco.

Foram aplicadas duas modalidades de questionário: uma após cada palestra informativa para avaliar a aceitação, compreensão e importância do trabalho, e outra nos domicílios para levantamento da prevalência e a qualidade do uso de medicamentos. Além disso, foram confeccionados folders informativos a respeito do tema para distribuição em cada atividade e nos domicílios visitados. Envolveram-se, como recursos humanos do trabalho, profissionais da Estratégia Saúde da Família (médicos, enfermeiros, agentes comunitários de saúde - ACS), equipe de enfermagem e de Saúde Bucal e profissionais do Núcleo de Apoio à Saúde da Família (NASF).

As variáveis em estudo foram: número de famílias, microárea de residência, número de membros em cada família, idade, sexo e número de medicamentos utilizados por cada 
membro da família no momento da enquete. Além disso, foram avaliados também a idade, o sexo e a escolaridade, o contato médico nos últimos 12 meses e a prática da automedicação do respondente que atendeu o ACS. O apoio do ACS foi fundamental para possibilitar a expansão da cobertura, principalmente nas áreas rurais ${ }^{15}$.

A análise das variáveis em questão teve o objetivo de verificar a existência de uma relação entre polifarmácia domiciliar, microárea de residência, sexo, idade, número de membros na família e escolaridade.

Resumidamente, as principais atividades consistiram em: palestra informativa e educativa para as equipes de saúde e a população; aplicação de questionários orientados para o uso racional de medicamentos; recolhimentos de medicamentos sem uso, sobras, data de validade vencida ou de qualidade duvidosa dos domicílios; avaliação e orientação profissional das famílias vulneráveis e identificação dos grupos de risco para consulta médica e avaliação clínica das funções renal e hepática.

Os critérios de inclusão foram participantes das reuniões e grupos terapêuticos, grupos de atividades do Projeto Agita Rio Fortuna, e a totalidade das famílias visitadas e que responderam espontaneamente aos questionários, em caráter de amostragem de conveniência. Critério de exclusão foram pessoas ausentes ou que não optaram pelo preenchimento dos questionários.

A abordagem conceitual consistiu no esclarecimento sobre "uso racional de medicamentos", "polifarmácia", "eventos e efeitos adversos", "efeitos colaterais", "automedicação", "interações medicamentosas", "intoxicação medicamentosa" e orientação de como evitar intoxicação por medicamentos.

O diagnóstico situacional relevante consistiu na existência de duas equipes de saúde da família, que abrangeram $100 \%$ da população essencialmente rural, de 4.646 habitantes (Instituto Brasileiro de Geografia e Estatística - IBGE, estimativa de 2010). Tal população foi distribuída em 11 microáreas, 1.278 famílias/residências (31), tendo 2.403 homens e 2.243 mulheres, nas faixas etárias menores de 20 anos (1.246), entre 20 e 59 anos (2.738) e maiores de 60 anos (662). Houve prevalência de 105,68 casos de hipertensos por cada 1.000 habitantes e 17,43 casos de diabetes por 1.000 habitantes. As cinco queixas mais comuns, que levaram a população a procurar a sua Unidade de Saúde em 2009, foram por doenças do aparelho cardiovascular, doenças do aparelho respiratório, doenças infectocontagiosas, doenças do aparelho digestivo, gravidez e puerpério, doenças osteomusculares e doenças neoplásicas. As principais causas de morbimortalidade, segundo a Sala de Situação do Ministério da Saúde (MS), foram: doenças cerebrovasculares e infarto do miocárdio; bronquite crônica e pneumonia; diabete mellitus; doença hipertensiva e neoplasias malignas. Do ponto de vista epidemiológico, ainda se destaca a marcante quantidade de pessoas que fazem uso de medicamentos controlados por problemas de saúde mental/psiquiátrico, sendo os transtornos de humor depressivo, a insônia e a ansiedade em sua grande maioria.

\section{Resultados e discussão}

Realizaram-se 13 palestras explicativas do trabalho, em diferentes ambientes comunitários, reunindo mais de 100 participantes. Os agentes comunitários de saúde realizaram visitas em 1.054 domicílios, dentre 1.278 famílias cadastradas no Município de Rio Fortuna, de agosto de 2010 a março de 2011.

Das atividades coletivas (Tabela 1), 98\% das pessoas acharam importantes as informações fornecidas, $1 \%$ não achou importante e $1 \%$ não respondeu a questão. Quanto à questão do entendimento subjetivo do conteúdo da exposição, $94 \%$ se sentiram mais orientados e $6 \%$ não se sentiram mais orientados. Em relação à automedicação, 50\% dos participantes referem já ter usado medicamentos sem receita médica, $46 \%$ não usaram medicamentos sem receita e $4 \%$ não responderam a questão. No momento da pesquisa, $20 \%$ das pessoas praticavam a automedicação, $76 \%$ não tomavam medicação sem receita e $4 \%$ não responderam. Por fim, $98 \%$ acharam importante realizar o presente trabalho sobre o uso racional de medicamentos e duas pessoas não responderam a questão.

Dos inquéritos domiciliares, foram realizadas visitas em 1.054 domicílios, dentre os quais 998 responderam aos ques-

Tabela 1. Percentual dos respondentes ao questionário avaliativo da palestra aplicada a 100 pessoas nas atividades coletivas, reuniões e grupos terapêuticos, realizados de setembro de 2010 a março de 2011, em Rio Fortuna.

\begin{tabular}{|c|c|c|c|}
\hline Questionário & $\operatorname{Sim}(\%)$ & Não (\%) & Não sei (\%) \\
\hline As informações fornecidas foram importantes? & 98 & 1 & 1 \\
\hline Você se sente mais orientado(a) a respeito do uso de medicamentos? & 94 & 6 & 0 \\
\hline Você já usou medicamentos sem receita médica? & 50 & 46 & 4 \\
\hline Você usa atualmente alguma medicação sem receita médica? & 20 & 76 & 4 \\
\hline Você acha importante realizar o trabalho do uso racional de medicamentos? & 98 & 0 & 2 \\
\hline
\end{tabular}


tionários. Destas, foram identificadas 185 famílias de risco quanto à presença de polifarmácia domiciliar (Tabela 2).

O consumo de medicamentos na população geral foi de zero a quatro comprimidos/família/dia (média=3,06), enquanto que, nas famílias em que havia polifarmácias, o consumo passou de 5 a 20 comprimidos por morador, por dia (média =9,24).

A média de membros do núcleo familiar foi de 3,42 pessoas, variando de morador único até nove pessoas. Nos últimos 12 meses, 90,6\% dos entrevistados realizaram alguma consulta médica ou tiveram contato com serviço médico, porém $58,1 \%$ afirmam ter, em algum momento, utilizado medicamentos sem receita médica.

Do grupo de risco (Tabela 3), em uso de polifarmácia domiciliar, $61,2 \%$ (123) eram mulheres com idade média de 66,25 anos, e $38,8 \%$ (78) eram homens com idade média de 62,68. O perfil das pessoas que atendem normalmente ao ACS nos domicílios (Tabela 4) é essencialmente de mulheres (84\%) com idade média de 43,81 anos (variando de 12 a 90 anos) e baixa escolaridade (60,7\% com Ensino Fundamental, muitos com até a terceira série realizada), sendo que $273(27,3 \%)$ das pessoas terminaram o Ensino Médio e apenas $110(11,0 \%)$ concluíram o Ensino Superior. O número maior de pessoas com Ensino Superior se encontrava nas microáreas da proximidade do centro do município, e o percentual de maior quantidade de uso de medicamentos por pessoa se encontrava nas microáreas do interior, com maior dificuldade de acesso ao centro de saúde.

A variável "Grau de Instrução" está baseada somente na menção dos três níveis de escolaridade, estando prejudicada a análise das séries completas ou incompletas.

Medicamentos recolhidos nas visitas domiciliares e os entregues espontaneamente na Unidade de Saúde totalizaram 4.651 espécimes, dentre comprimidos, soluções e pomadas, considerados sobras de tratamento, validade vencida, qualidade duvidosa ou simplesmente sem utilidade (Tabela 5, Gráfico 1).

Tabela 2. Características do consumo de medicamentos, em número de comprimidos (CPS), nas famílias visitadas e que responderam ao questionário dos Agentes Comunitários de Saúde, em suas respectivas microáreas, de setembro de 2010 a março de 2011, em Rio Fortuna.

\begin{tabular}{lcc} 
Variáveis & $\mathrm{n}$ & $\%$ \\
\hline Número de famílias respondentes & 998 & 100,0 \\
\hline Consulta médica nos últimos 12 meses & 904 & 90,6 \\
Uso de medicação sem receita & 580 & 58,1 \\
Número de famílias de risco & 185 & 18,5 \\
Número CPS/Família (média) & 3,06 & \\
Número CPS/Família no grupo de risco (média) & 9,24 & \\
Número dos membros por família (média) & 3,42 & \\
\hline
\end{tabular}

Tabela 3. Características do grupo de risco, identificado como famílias que possuem pelo menos um indivíduo usuário de cinco ou mais medicamentos por dia, questionadas nas visitas do Agente Comunitário de Saúde, de setembro de 2010 a março de 2011, no município de Rio Fortuna.

\begin{tabular}{lrrc}
\hline Grupo de risco & \multicolumn{1}{c}{$\mathrm{n}$} & $\%$ & Média de idade (anos) \\
\hline Mulher & 123 & 61,2 & 66,25 \\
Homem & 78 & 38,8 & 62,68 \\
Total & 201 & 100,0 & 64,46 \\
\hline
\end{tabular}

Tabela 4. Perfil do respondente, pessoas que comumente atenderam à visita do Agente Comunitário de Saúde, de setembro de $2010 \mathrm{a}$ março de 2011, em Rio Fortuna.

\begin{tabular}{lcc}
\hline Respondente & $\mathrm{n}$ & $\%$ \\
\hline Mulher & 837 & 84,0 \\
Homem & 159 & 16,0 \\
\hline Média de idade (mínimo-máximo) anos & $43,81(12-90)$ & \\
\hline Grau de instrução & $\mathrm{n}$ & $\%$ \\
\hline Ensino Fundamental & 606 & 60,7 \\
Ensino Médio & 273 & 27,3 \\
Ensino Superior & 110 & 11,0 \\
Não houve resposta & 9 & 1,0 \\
\hline
\end{tabular}

Tabela 5. Classificação e quantidade de medicamentos, em número de comprimidos (CPS) recolhidos nas visitas domiciliares, de setembro de 2010 a março de 2011, em Rio Fortuna.

\begin{tabular}{lc}
\hline Medicamentos recolhidos & Número de CPS \\
\hline Diabetes & 1.811 \\
Cardiovascular & 1.119 \\
AINE & 653 \\
\hline Psicotrópicos & 362 \\
Antibióticos & 259 \\
Gastrintestinal & 177 \\
Vitaminas & 120 \\
\hline Outros & 77 \\
Aparelho respiratório & 73 \\
\hline Total & 4.651 \\
\hline
\end{tabular}

AINE: anti-inflamatório não esteroide.

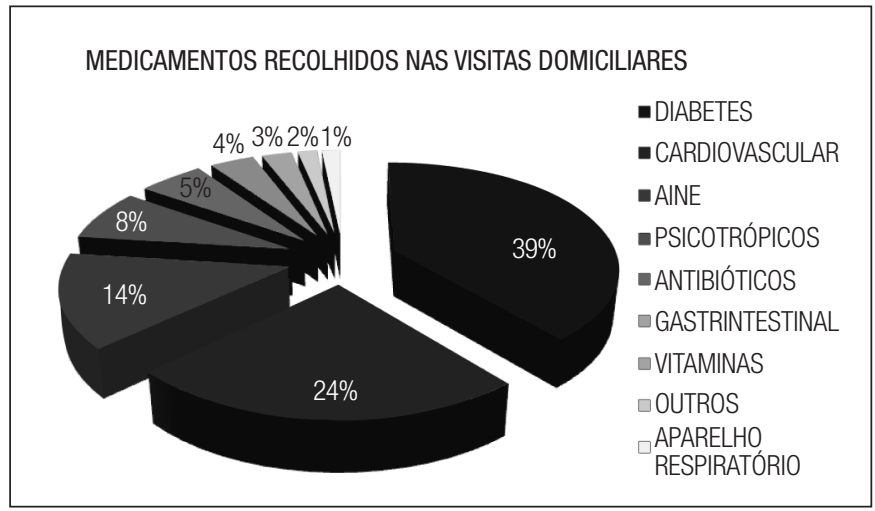

Gráfico 1. Percentual dos medicamentos recolhidos de agosto de 2010 a março de 2011, em Rio Fortuna. 
As cinco principais classes farmacológicas mais encontradas nos estoques domiciliares foram: medicamentos para diabetes, doenças cardiovasculares, anti-inflamatórios não-esteroides (AINE), psicotrópicos e antibióticos, em grande parte fornecidas pela Secretaria Municipal de Saúde.

\section{Considerações finais}

O Plano Nacional de Medicamentos e as ações governamentais ainda se mostram insuficientes para dar resposta adequada às necessidades da população no que diz respeito, principalmente, à oferta de insumos conforme quadro epidemiológico. Em muitos momentos, a população faz tratamento medicamentoso sem orientação profissional e poucos conhecem os riscos da automedicação e dos perigos das interações medicamentosas. No entanto, muito tem se avançado para efetivar o direito da assistência farmacêutica integral, por meio de instrumentos legais e normativos para garantir a disponibilidade, o acesso e o uso racional de medicamentos à população.

Além disso, a facilidade de acesso aos medicamentos tem gerado, em algumas situações, o consumo exagerado e até mesmo descontrolado de fármacos, resultando em abusos, intoxicações e exposição às interações e aos efeitos adversos. Outro fator que agrava o problema do uso de medicamentos é o aumento da expectativa de vida e a longevidade da população com múltiplas patologias. Estima-se que $23 \%$ da população brasileira consomem $60 \%$ da produção nacional de medicamentos, principalmente as pessoas acima de $60 \operatorname{anos}^{16}$. Há evidências de que os idosos norte-americanos, vivendo na comunidade, ingerem, em média, de 2,7 a 4,2 medicamentos por dia ${ }^{17}$. Marin et al. ${ }^{18}$ consideram que os idosos que utilizam múltiplos medicamentos são propensos à maior vulnerabilidade nas condições de saúde e que, em média, pessoas que usam cinco ou mais medicamentos revelam 5,2 diagnósticos, por idoso. Tais diagnósticos revelam a necessidade de medidas envolvendo mudanças no estilo de vida, além do acompanhamento sistemático dessas pessoas.

Da mesma forma, o presente trabalho permite algumas inferências a respeito do consumo de medicamentos de acordo com a faixa etária, o gênero e o grau de escolaridade. O perfil das pessoas que possuem polifarmácias domiciliares e que mais consomem medicamentos no município de Rio Fortuna são mulheres $(61,0 \%)$ de idade avançada (média de 66,25 anos), morando no interior e com pouca escolaridade. Não foram verificadas as classes farmacológicas mais comumente utilizadas pelas famílias entrevistadas, mas houve recolhimento de medicamentos considerados sem uso, sobras de tratamentos ou com data de validade vencida. Este trabalho de orientação e informação à população é permanente, e as pessoas que fazem uso de polifarmácia identificadas pelas visitas serão continuamente acompanhadas para que sejam evitados possíveis danos por excesso de medicamento ou por possível interação entre fármacos.

A quantidade de medicamentos para uso em diabéticos e portadores de doenças cardiovasculares, com data de validade vencidos, permite refletir sobre o acesso e o não-uso desses medicamentos por parte da população.

Trabalhos sobre o perfil do consumo de medicamentos podem também contribuir para a discussão sobre a problemática do acesso da população às terapias farmacológicas e podem subsidiar políticas públicas que visem promover o acesso universal e o uso racional dos medicamentos.

Portanto, atuação multiprofissional na atenção em saúde é essencial para o constante aprimoramento do cuidado da saúde da população. O entendimento da necessidade da educação permanente em saúde, tanto dos profissionais quanto da população e da gestão setorial, pode promover grandes mudanças no paradigma assistencial.

\section{Referências}

1. Ministério da Saúde. Secretaria de Políticas de Saúde. Política Nacional de Medicamentos [Informe Técnico Institucional]. Rev Saúde Pública. 2000; 34(2): 206-9. [Internet] [acesso em 2011 Mar 18]. Disponível em: http://www.scielo.br/pdf/rsp/v34n2/1960.pdf

2. Alves TNP. Política Nacional de Medicamentos: análise a partir do contexto, conteúdo e processos envolvidos [Tese]. Rio de Janeiro: Universidade do Estado do Rio de Janeiro; 2009189 p. [Internet] [acesso em 2011 Mar 14]. Disponível em: http://www.tesesims.uerj.br/lildbi/ docsonline/pdf/alves_terezinha.pdf

3. Pelicioni AF. Padrão de consumo de medicamentos em duas áreas da Região Metropolitana de São Paulo, 2001-2002 [Tese]. São Paulo: Universidade de São Paulo. Faculdade de Saúde Publica; Departamento de Epidemiologia; 2005. 104 p.

4. Oliveira MA, Bermudez JAZ, Osório-de-Castro CGS. Assistência farmacêutica e acesso a medicamentos. Rio de Janeiro: Fiocruz; 2007. $112 \mathrm{p}$.

5. WHO. Global partnerships for health. WHO Drug Information. 1999; 13(2): 61-4. [Acesso em 2011 Abr 14]. Disponível em: http://apps.who. int/medicinedocs/documents/s14172e/s14172e.pdf

6. Aquino DS. Por que o uso racional de medicamentos deve ser uma prioridade? Ciênc saúde coletiva. 2008; 13 Suppl:733-6. [Internet] [acesso em 2011 Abr 14]. Disponível em: http://www.scielo.br/scielo. php?script $=$ sci_arttext\&pid=S1413-81232008000700023\&lng=pt http://dx.doi.org/10.1590/S1413-81232008000700023.

7. Fernandes LC. Caracterização e análise da farmácia caseira ou estoque domiciliar de medicamentos [resumo de dissertação]. Porto Alegre: Faculdade de Farmácia, Universidade Federal do Rio Grande do Sul; 2000. [Internet] [acesso em 2011 Mar 20]. Disponível em: http://www. ufrgs.br/farmacia/cadfar/v17n1/resumos/cadfarv17n1P46.html

8. Ribeiro MA, Heineck I. Estoque domiciliar de medicamentos na comunidade ibiaense acompanhada pelo Programa Saúde da Família, em Ibiá-MG, Brasil. Saúde Soc. 2010; 19(3): 653-63. [Internet] [acesso em 2011 Mar 20]. Disponível em: http://www.scielo.br/pdf/sausoc/ v19n3/16.pdf. http://dx.doi.org/10.1590/S0104-12902010000300016 
9. Brasil. Ministério da Saúde. Secretaria de Ciência, Tecnologia e Insumos Estratégicos. Departamento de assistência Farmacêutica e de Insumos Estratégicos. Formulário Terapêutico Nacional 2008- Rename 2006. (série B. Textos Básicos em saúde). Brasília, DF: Ministério da Saúde; 2008. 897p.

10. Carvalho MFC. A polifarmácia em idosos no Município de São Paulo Estudo SABE - saúde, bem-estar e envelhecimento [dissertação]. São Paulo: Escola de Saúde Pública; 2007. 195 p.

11. Ferreira MBC. Uso Racional de Medicamentos [apresentação do I Congresso Brasileiro de Uso Racional de Medicamentos]. Departamento de Farmacologia da Universidade Federal do Rio Grande do Sul. Porto Alegre; 2005. [Internet] [acesso em 2011 Mar 14]. Disponível em: http:// www.anvisa.gov.br/divulga/eventos/propaganda_medicamentos/ seminarios/regional_sul/uso_racional_medicamentos.pdf

12. Flores LM, Mengue SS. Uso de medicamentos por idosos em região do sul do Brasil. Rev Saúde Pública. 2005; 39(6): 924-9. [Internet]. Disponível em: http://www.scielo.br/pdf/rsp/v39n6/26987.pdf http:// dx.doi.org/10.1590/S0034-89102005000600009

13. Vilarino JF, Soares IC, Silveira CM, Rödel APP, Bortoli R, Lemos RR. Perfil da automedicação em município do Sul do Brasil. Rev Saúde Pública. 1998; 32(1): 43-9. [Internet] [acesso em 2011 Mar 14]. Disponível em: http://www.scielosp.org/pdf/rsp/v32n1/2390.pdf http://dx.doi. org/10.1590/S0034-89101998000100006
14. CIT/SC. Centro de Informações Toxicológicas de Santa Catarina. Estatísticas Gerais e Anuais, do período de maio de 1984 a dezembro de 2010. [Internet] [acesso em 2011 Mar 14]. Disponível em: www.cit. sc.gov.br

15. Silva JA. O agente comunitário de saúde do projeto QUALIS: agente institucional ou agente de comunidade [Tese]. São Paulo: Faculdade de Saúde Pública, Universidade de São Paulo; 2001. 231 .

16. Teixeira JJ, Lefèvre F. A prescrição medicamentosa sob a ótica do paciente idoso. Rev Saúde Pública. 2001; 35(2): 207-13. http://dx.doi. org/10.1590/S0034-89102001000200016

17. Hanlon JT, Schmader KE, Ruby CM, Weinberger M. Suboptimal prescribing in older inpatients and outpatients. J Am Geriatr Soc. 2001; 49(2): 200-9. [Internet] [acesso em 2011 Mar 14]. Disponível em: https://courses.washington.edu/pharm550/Week1/reading\%20 wk1\%20Hanlon\%20Schmaeder.pdf. http://dx.doi.org/10.1046/j.15325415.2001.49042.x

18. Marin MJS, Rodrigues LCR, Druzian S, Cecílio LCO. Diagnósticos de enfermagem de idosos que utilizam múltiplos medicamentos. Rev Esc Enferm. 2010; 44(1): 47-52. Extraído do projeto "Os idosos precisam de cuidados especiais: uma proposta para planejamento das ações em uma Unidade de saúde da Família"; 2006. [Internet] [acesso em 2011 Mar 18]. Disponível em: http://www.scielo.br/pdf/reeusp/v44n1/a07v44n1. pdf http://dx.doi.org/10.1590/S0080-62342010000100007 